\title{
Avaliação da Experiência do Usuário e da Usabilidade de Aplicativos para Prática de Exercícios Físicos: Um Mapeamento Sistemático da Literatura
}

\author{
Josué V. Ferreira ${ }^{1}$, Viviane A. Santos ${ }^{1}$, Carlos S. Portela ${ }^{1}$ \\ ${ }^{1}$ Programa de Pós-graduação em Computação Aplicada - Universidade Federal do Pará \\ Tucuruí - PA - Brasil

\begin{abstract}
During the COVID-19 pandemic, there was a huge increase in the use of apps to perform physical exercises. In this context, this paper presents the results of a Systematic Mapping of Literature (SML) of studies that report the evaluation of Usability and User Experience of applications intended for physical exercises, in order to recognize which methods are most suitable for the identifying and selecting a tool to help users in achieving their goals: weight loss, lean mass gain, etc. The results show that $42 \%$ of the studies use the SUS questionnaire, 14\% use the think aloud method, only $7 \%$ the cognitive walkthrough, and $37 \%$ other methods.
\end{abstract}

Resumo. Durante a pandemia da COVID-19, houve um grande aumento do uso de aplicativos para a realização de exercícios físicos. Neste contexto, este trabalho apresenta os resultados de um Mapeamento Sistemático da Literatura (MSL) de trabalhos que relatam a avaliação de Usabilidade e da Experiência do Usuário de aplicativos destinados à exercícios físicos, a fim de reconhecer quais métodos são mais adequados para que o usuário identifique e selecione uma ferramenta que o auxilie a atingir seus objetivos: perda de peso, ganho de massa magra, etc. Os resultados apontam que $42 \%$ dos estudos adotam o questionário SUS, 14\% usam o método think aloud, apenas $7 \%$ a técnica cognitive walkthrough e $37 \%$ outros métodos.

\section{Introdução}

A constante prática de exercícios físicos promove ao indivíduo benefícios à saúde física, mental e social, ajudando a prevenir inúmeras enfermidades, como diabetes tipo 2, doenças cardiovasculares, obesidade, etc. [Lear et al. 2017]. Desta forma, atividades físicas regulares potencializam a aceleração do metabolismo em pessoas sedentárias, aumentam a queima de calorias e, juntamente com uma alimentação adequada, possibilitam ao praticante um estilo de vida mais saudável e bem-estar [Kyu et al. 2016].

Apesar dos benefícios, muitas pessoas não praticam nenhum tipo de atividade física, seja por falta de tempo, desmotivação e/ou carência de conhecimento necessário. Existem também situações atípicas como a pandemia do novo coronavírus (SARSCOV-2), em que foram impostas medidas sanitárias restritivas pelos órgãos de saúde pública, havendo a necessidade das pessoas se manterem isoladas em suas casas [Aquino et al. 2020]. Neste cenário, as academias, parques e praças tiveram que ser fechados temporariamente nos períodos de maior agravamento da COVID-19. 
Aplicativos móveis se apresentam como uma alternativa para realização de atividades físicas fora das academias [Romeo et al. 2019]. A interface desses aplicativos direcionados à praticantes de atividades físicas constitui a parte com a qual o usuário mantém maior contato [Almeida et al. 2016]. Sendo assim, critérios de qualidade são muito importantes para esses aplicativos, já que a experiência do usuário é fundamental para que os exercícios físicos sejam bem executados, consequentemente, o praticante não adquira algum tipo de lesão a curto ou longo prazo. Além disso, deve-se atentar para a interface dessas aplicações, pois uma usabilidade ruim pode tornar a aplicação com pouca ou nenhuma utilidade. A interface proporciona um grande impacto sobre os usuários e interferem no seu comportamento físico e psicológico [Jones et al. 2016].

Desta maneira, este trabalho apresenta os resultados de um Mapeamento Sistemático da Literatura (MSL) de trabalhos que relatam a avaliação de Usabilidade e da Experiência do Usuário (do inglês User Experience - UX) de aplicativos destinados a exercícios físicos, a fim de reconhecer quais métodos são mais adequados para que o usuário identifique e selecione uma ferramenta que o auxilie a atingir seus objetivos: perda de peso, ganho de massa magra, qualidade de vida, etc.

Além dessa seção introdutória, a Seção 2 apresenta a fundamentação teórica acerca da usabilidade e UX. Na Seção 3 é apresentado o Mapeamento Sistemático da Literatura. Na Seção 4, são destacados os resultados obtidos, de acordo com as questões de pesquisa. Por fim, a Seção 5 apresenta as considerações finais.

\section{Fundamentação}

Segundo a norma ISO/IEC 25010 (2011), a usabilidade é definida como a "capacidade do produto de software de ser compreendido, aprendido, operado e atraente ao usuário, quando usado sob condições específicas". A norma ISO 9421-210 define a UX como "a percepção e as respostas de uma pessoa resultantes do uso ou da antecipação do uso de um produto". Neste sentido, a usabilidade abrange diretrizes voltadas às funcionalidades do software enquanto ferramenta (eficácia e eficiência) para execução de tarefas pelo usuário. Já a UX, remete a fatores mais subjetivos, de como o usuário se sente antes, durante e após o uso de um produto, suas experiências, emoções e demais aspectos significativos e preciosos [Vermeeren et al. 2010].

Dessa maneira, quando se realiza a avaliação de UX e Usabilidade, pode-se mensurar os sentimentos e níveis de satisfação do usuário/cliente em relação à aplicação (UX), assim como a capacidade de executar tarefas específicas na aplicação (usabilidade) [Väätäjä, Koponen e Roto 2009]. Assim, as avaliações de usabilidade e experiência do usuário, principalmente durante a fase de desenvolvimento, são essenciais para o sucesso e aceitação do produto por parte dos usuários [Wicahyono et al. 2019].

Em [Baig et al. 2020] testaram o protótipo do aplicativo mHealth com um grupo de usuários. Diante disso, foi possível validar a usabilidade da ferramenta (design, recursos e funcionalidades) visando atender aos objetivos e necessidades dos médicos, enfermeiros e pacientes, uma vez que ela fosse implementada. Neste sentido, as avaliações de usabilidade, realizadas através de questionários e técnicas de inspeção, possibilitaram um feedback do usuário muito útil, que permitiram contornar possíveis erros na aplicação, dispondo satisfação e uma boa experiência de uso (UX). 


\section{Metodologia}

O Mapeamento Sistemático da Literatura é um método que realiza uma ampla revisão de estudos primários, possibilitando a obtenção de destaques em uma determinada área de pesquisa, para direcionar e fornecer uma quantidade de fatos identificados sobre a mesma. O mapeamento realizado nesta pesquisa foi baseado no processo descrito por [Petersen, Vakkalanka e Kuzniarz 2015], que definem cinco etapas fundamentais a serem seguidas: 1. Definição de questões de pesquisa; 2. Realização da pesquisa de estudos primários relevantes; 3. Seleção dos estudos; 4. Identificação de palavras-chaves nos títulos e resumos; e 5. Extração de dados e mapeamento dos estudos.

A fim de levantar os estudos desse mapeamento, foram realizadas buscas nas seguintes bases: ACM Digital Library (DL), Science Direct e PubMed. O banco de dados da ACM DL é um repositório renomado que inclui publicações na área da computação, contendo publicações de editoras acadêmicas, sendo uma base de alta relevância para profissionais de computação [ACM 2021]. Já a base de dados Science Direct, abrange publicações científicas de periódicos e livros no âmbito da saúde, e a PubMed abrange literatura biomédica e de ciências da vida, através de periódicos de ciências biológicas e livros online [PubMed 2021].

\subsection{Planejamento do Mapeamento Sistemático}

O objetivo deste MSL consiste em obter uma visão geral das métricas de usabilidade e UX usadas para avaliar aplicativos voltados para exercícios físicos, no período de 5 anos (janeiro de 2016 a dezembro de 2020). O levantamento aconteceu entre janeiro e março de 2021. A fim de atender esse objetivo, foram definidas as seguintes Questões de Pesquisa (QP): QP1: Quais métodos foram utilizados na avaliação dos aplicativos de exercício físico?; QP2: Qual o perfil do público-alvo desses aplicativos?; QP3: $\quad$ Em qual etapa do desenvolvimento de software o aplicativo foi avaliado?

Para definir a string de busca do mapeamento, foram realizadas pesquisas no Google acadêmico com o intuito de identificar pesquisas que continham palavras-chave e sinônimos relacionados a métodos de avaliação de aplicativos, saúde, exercícios físicos, dentre outros. Desta forma, obteve-se as seguintes palavras-chave: "mobile app", "physical exercise", "physical activity", "fitness", "user experience", “ux", "usability", "user satisfaction" e "evaluation method". A string de busca final resultou em: ("mobile app") AND ("Physical Exercise" OR "Physical Activity" OR "Fitness") AND ("User Experience" OR "UX" OR "Usability" OR "User Satisfaction" OR "Evaluation Method").

Em seguida, a fim de filtrar os estudos selecionados pela string de busca, foram definidos os Critérios de Inclusão (CI) e os Critérios de Exclusão (CE): CI1: Os estudos devem estar escritos em inglês; CI2: Os estudos devem relatar o desenvolvimento ou uso de aplicativos para exercícios físicos; CI3: Os estudos devem avaliar a usabilidade e a experiência do usuário de aplicativos móveis; CI4: Os estudos devem estar disponíveis na web para download; CI5: Os estudos devem ser atuais, publicados nos últimos 5 anos (2016-2020); CE1: Os estudos que estejam duplicados nas diferentes bases; CE2: Relatórios técnicos ou documentos que estão disponíveis em formato de resumos ou apresentações; CE3: Estudos secundários (revisões sistemáticas da literatura e mapeamentos). 


\subsection{Estudos Selecionados}

Após o uso da string de busca nos motores de busca, retornaram 263 estudos. Aplicados os CE e CI, foram selecionadas 20 pesquisas para análise, conforme Quadro 1.

Quadro 1. Distribuição de artigos selecionados nos mecanismos de busca

\begin{tabular}{|l|c|c|c|c|}
\hline Base & $\begin{array}{l}\text { Estudos } \\
\text { retornados }\end{array}$ & $\begin{array}{l}\text { Após critérios de } \\
\text { exclusão }\end{array}$ & $\begin{array}{l}\text { Após critérios de } \\
\text { inclusão }\end{array}$ & $\begin{array}{l}\text { Após leitura } \\
\text { completa }\end{array}$ \\
\hline ACM DL & 36 & 1 & 1 & 0 \\
\hline PubMed & 35 & 18 & 16 & 12 \\
\hline Science Direct & 192 & 3 & 3 & 2 \\
\hline Total & $\mathbf{2 6 3}$ & $\mathbf{2 2}$ & $\mathbf{2 0}$ & $\mathbf{1 4}$ \\
\hline
\end{tabular}

Após a aplicação dos critérios, com 12 estudos, a base da PubMed contribuiu para a maior parte da seleção, haja vista que, esse banco de dados engloba inúmeras pesquisas na área da medicina. Enquanto que na Science Direct, apesar do maior número de artigos retornados na busca inicial, apenas 2 atenderam aos critérios. Dessa maneira, os CI2 e CI3 definiram a exclusão dos outros 6 artigos. Portanto, a seleção final foi constituída de 14 estudos, que apresentavam um ou mais métodos de avaliação, seja de usabilidade e/ou UX, conforme é apresentado no Quadro 2.

\section{Quadro 2. Identificação dos estudos levantados (ordem alfabética)}

\begin{tabular}{|c|c|}
\hline Título & Autores \\
\hline $\begin{array}{l}\text { A Mobile App to Promote Adapted Exercise and Social Networking for } \\
\text { People With Physical Disabilities: Usability Study }\end{array}$ & Lai et al. 2019 \\
\hline $\begin{array}{l}\text { A Personalized Physical Activity Coaching App for Breast Cancer Survivors: } \\
\text { Design Process and Early Prototype Testing }\end{array}$ & $\begin{array}{l}\text { Monteiro-Guerra } \\
\text { et al. } 2020\end{array}$ \\
\hline $\begin{array}{l}\text { Continuous use of fitness apps and shaping factors among college students: A } \\
\text { mixed-method investigation }\end{array}$ & Zhang e Xu 2020 \\
\hline $\begin{array}{l}\text { Development of a Weight Loss Mobile App Linked With an Accelerometer } \\
\text { for Use in the Clinic: Usability, Acceptability, and Early Testing of its Impact } \\
\text { on the Patient-Doctor Relationship }\end{array}$ & Choo et al. 2016 \\
\hline $\begin{array}{l}\text { Efficacy of a Mobile Social Networking Intervention in Promoting Physical } \\
\text { Activity: Quasi-Experimental Study }\end{array}$ & Tong et al. 2019 \\
\hline $\begin{array}{l}\text { Evaluating the Impact of the HeartHab App on Motivation, Physical Activity, } \\
\text { Quality of Life, and Risk Factors of Coronary Artery Disease Patients: } \\
\text { Multidisciplinary Crossover Study }\end{array}$ & $\begin{array}{l}\text { Sankaran, } \\
\text { Dendale e Coninx } \\
2019\end{array}$ \\
\hline $\begin{array}{l}\text { Experiences and Perceptions of Adults Accessing Publicly Available Nutrition } \\
\text { Behavior-Change Mobile Apps for Weight Management }\end{array}$ & $\begin{array}{l}\text { Lieffers et al. } \\
2018\end{array}$ \\
\hline $\begin{array}{l}\text { Mobile and Wearable Device Features that Matter in Promoting Physical } \\
\text { Activity }\end{array}$ & Wang et al. 2016 \\
\hline $\begin{array}{l}\text { MOVE: A Mobile App Designed for Social Health Relations in Residential } \\
\text { Areas }\end{array}$ & $\begin{array}{l}\text { Kanstri } \\
2018\end{array}$ \\
\hline $\begin{array}{l}\text { PuzzleWalk: A theory-driven iterative design inquiry of a mobile game for } \\
\text { promoting physical activity in adults with autism spectrum disorder }\end{array}$ & Kim et al. 2020 \\
\hline $\begin{array}{l}\text { The Development of an Arabic Weight-Loss App Akser Waznk: Qualitative } \\
\text { Results }\end{array}$ & $\begin{array}{l}\text { Alturki e Gay } \\
2019\end{array}$ \\
\hline $\begin{array}{l}\text { Tracking Valued and Avoidant Functions with Health Behaviors: A } \\
\text { Randomized Controlled Trial of the Acceptance and Commitment Therapy } \\
\text { Matrix Mobile App }\end{array}$ & Levin et al. 2020 \\
\hline $\begin{array}{l}\text { Usability inquiry of a gamified behavior change app for increasing physical } \\
\text { activity and reducing sedentary behavior in adults with and without autism } \\
\text { spectrum disorder }\end{array}$ & Lee et al. 2020 \\
\hline VA FitHeart, a Mobile App for Car & Beatty et al. 2018 \\
\hline
\end{tabular}




\section{Resultados}

\subsection{Síntese dos Estudos}

Em [Lai et al. 2019] testaram a usabilidade de um aplicativo fitness $m$ Health inclusivo (denominado SUPER-HEALTH), desenvolvido especialmente para Pessoas com Deficiência (PcD). Este estudo foi composto de duas etapas: teste de usabilidade e uma entrevista. A entrevista buscou avaliar 4 componentes quantitativos e qualitativos, sendo a eficácia e eficiência do aplicativo (quantidade) e, a satisfação e a utilidade (qualidade). Neste sentido, os critérios de eficácia e eficiência correspondem à usabilidade, ou seja, relacionados às funcionalidades do software, enquanto que a utilidade e satisfação, são características pertinentes à experiência do usuário, remetendo à sentimentos e emoções dos usuários. Para avaliar os 4 componentes, foi necessário incluir 7 critérios (teste de usabilidade), sendo eles: abrir o aplicativo; localizar e abrir um papel; localizar e abrir um crachá; localizar, abrir e criar uma postagem de uma palavra no feed de notícias; adicionar um usuário como amigo; localizar e visualizar a tabela de classificação; reproduzir os vídeos; e realizar a rotina de exercícios adaptada.

O trabalho de [Beatty et al. 2018] avaliou a usabilidade de um aplicativo móvel (VA FitHeart) de atividade física e medidas de saúde para pacientes em Recuperação Cardíaca (RC). Durante o desenvolvimento da pesquisa, a ferramenta passou por atualizações em sua interface de acordo com o feedback fornecido pelos participantes dos testes de usabilidade. A avaliação de usabilidade utilizada com os participantes foi a Escala de Usabilidade do Sistema (SUS) (escala de 0 a 100). Considerou-se a pontuação maior que 70 uma usabilidade aceitável. O SUS é um questionário com um conjunto de 10 afirmações, capaz de identificar a opinião dos usuários sobre a usabilidade de um sistema [Sauro 2011].

O objetivo de [Tong et al. 2019] foi testar um aplicativo móvel (fit.healthy.me) de rede social, conectado a um rastreador vestível para monitorar a contagem de passos diários e, consequentemente, promover a realização de atividade física para pessoas menos ativas e com sobrepeso. Uma das etapas do projeto investigou o envolvimento dos usuários e a usabilidade do aplicativo. Com uma proposta similar, [Monteiro-Guerra et al. 2020] avaliaram a usabilidade de um protótipo de aplicativo de caminhada para pacientes em pós-tratamento do câncer de mama. O questionário SUS foi utilizado em ambas as pesquisas. Em [Levin et al. 2020] também usaram o SUS para testar a usabilidade de um aplicativo mHealth, desenvolvido após um estudo clínico randomizado, que visou estimular o automonitoramento da atividade física $\mathrm{e}$ alimentação adequada em indivíduos que têm interesse em manter hábitos saudáveis.

Em [Lee et al. 2020] observaram que técnicas de gamificação têm grande potencial para estimular a atividade física em adultos com Transtorno do Espectro Autista (TEA). Neste sentido, realizaram a avaliação de usabilidade (por meio do questionário SUS) de um aplicativo móvel gamificado (PuzzleWalk), a afim de aumentar a atividade física e reduzir o sedentarismo em adultos com e sem TEA.

O trabalho de [Alturki e Gay 2019] realizou entrevistas semiestruturadas de usabilidade de dois aplicativos móveis para perda de peso (Twazon e Aded Surat), com o objetivo de coletar o feedback dos participantes dos testes (sugestões, pontos fortes e fracos). Foi relatado que esses apps tinham baixa usabilidade e pouca aderência às 
particularidades do público-alvo. Portanto, o estudo propôs um aplicativo móvel (Akser Waznk) para atividade física e nutrição adequada direcionados a usuários com sobrepeso, visando atender suas especificidades. Os usuários também testaram a usabilidade do Akser Waznk, enfatizando o seu design interativo e os recursos motivacionais são fáceis de usar.

Em [Sankaran, Dendale e Coninx 2019] avaliaram o HeartHab, um app que visa motivar os pacientes a manter uma rotina de exercícios físicos, aumentando os níveis de qualidade de vida e bem-estar dos pacientes. A telereabilitação é definida como o método em que se utilizam tecnologias de comunicação para prover reabilitação à distância [Kairy et al. 2009], e tem sido muito eficaz no tratamento da Doença Arterial Coronariana (DAC), auxiliando os pacientes a se reabilitarem em casa. Para avaliar a experiência do usuário do aplicativo, foi realizada uma entrevista semiestruturada abordando os seguintes aspectos dos usuários: como o aplicativo promoveu a ser mais ativo fisicamente; a adoção de um estilo de vida mais saudável; suporte fornecido em sua reabilitação e o uso contínuo do app.

O estudo de [Kanstrup et al. 2018] avaliou a versão beta de um aplicativo (MOVE) que apoia a prática de exercícios físicos em pessoas com alto risco de mortalidade (obesos, inativos, fumantes e indivíduos com doenças mentais e crônicas). A técnica think aloud (ou pensar em voz alta) [Fonteyn, Kuipers e Grobe 1993], é uma metodologia fácil e eficiente, sendo amplamente empregada nos testes de usabilidade, permitindo verbalizar as impressões dos usuários a respeito da interface. $\mathrm{O}$ instrumento de avaliação de usabilidade adotado foi um questionário focado na experiência do usuário (perguntas sobre exercícios físicos e saúde móvel) e o teste think aloud.

Em [Wang et al. 2016] desenvolveu-se um dispositivo (Fitbit One), composto por um rastreador, site, prompts baseados em SMS e um aplicativo móvel, a fim de motivar a mudança de comportamento e promover a atividade física em pessoas obesas ou com sobrepeso. A usabilidade do aplicativo foi avaliada por meio de um questionário perguntando aos usuários sobre sua experiência com o produto, por exemplo, se era fácil de usar, se ajudava a ser mais ativo fisicamente, entre outros.

O uso de um aplicativo móvel pode ser uma solução eficaz para auxiliar a prática de exercícios físicos e reduzir a ansiedade em adultos com TEA. Então, [Kim et al. 2020] realizaram um estudo de viabilidade do PuzzleWalk, um protótipo de alta fidelidade, que promove a atividade física (principalmente a caminhada) com jogos desenvolvidos para adultos com autismo. A usabilidade foi baseada no protocolo think aloud, no método cognitive walkthrough e questionário SUS. O cognitive walkthrough (ou acompanhamento cognitivo) [Polson et al. 1992], é um método para mensurar a percepção dos usuários sobre a usabilidade de um sistema através da escala Likert de 5 pontos, após a conclusão de cada etapa do projeto.

Em [Choo et al. 2016] desenvolveram um aplicativo móvel vinculado a um acelerômetro para um programa de perda de peso em um ambiente clínico e, posteriormente, foi realizada a avaliação de usabilidade mediante a questionários e análise de dados. Além disso, a avaliação de aceitabilidade expressou a experiência geral dos usuários com relação ao uso do aplicativo, sua confiança percebida nas informações disponibilizadas e sua satisfação com a experiência. Os testes de usabilidade mediram a eficácia, questionando se os usuários conseguiam realizar as 
tarefas de forma fácil, sem cometer erros. Neste sentido, a avaliação do aplicativo foi dividida em duas etapas: a primeira foi pela média de login (o número médio de logins no aplicativo por dia), que expressava a frequência que o participante usava o aplicativo durante o dia do teste; e, a outra, deu-se através do uso de cada módulo específico (o número de cliques por módulo dividido pelo número total de cliques durante o período da pesquisa).

A pesquisa de [Zhang e $\mathrm{Xu}$ 2020] explorou as causas psicológicas que determinam a intenção de continuidade de estudantes universitários em usar aplicativos de condicionamento físico. Os autores utilizaram um método misto explicativo sequencial de design, composto por uma pesquisa quantitativa, com o objetivo de identificar fatores que determinam a intenção do uso contínuo do app. Para isso, foi disponibilizado um questionário para saber a opinião e experiência do usuário (por meio da autoavaliação). Ademais, foi realizada uma pesquisa qualitativa, utilizando entrevistas semiestruturadas.

As entrevistas semiestruturadas também foram adotadas na pesquisa de [Lieffers et al. 2018], para medir a experiência anterior/atual dos usuários com relação à nutrição e aplicativos móveis para gerenciamento de peso. Segundo os autores, pouco se sabe sobre as experiências e percepções dos usuários de aplicações de suporte à nutrição, quando usadas fora de um ambiente de teste de pesquisa.

\subsection{Métodos para Avaliação de Aplicativos de Exercícios Físicos}

A QP1 buscava identificar quais métodos foram utilizados na avaliação dos aplicativos de exercícios físicos. Os resultados são apresentados na Figura 1.

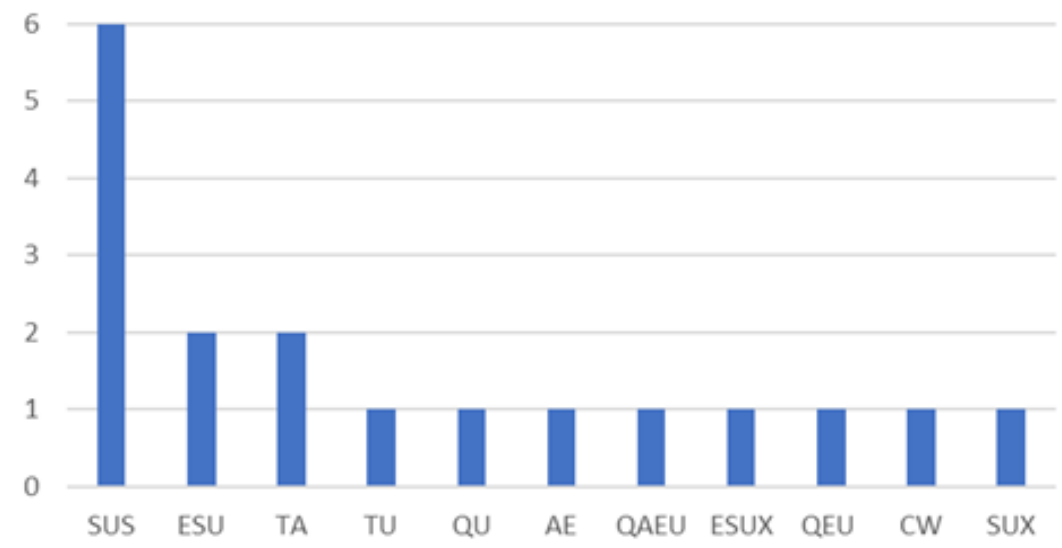

Figura 1. Métodos de Avaliação encontrados

Presente em 6 estudos, a escala SUS foi o método mais utilizado pelos autores. Essa metodologia tem sido vastamente empregada para avaliar a usabilidade em estudos comerciais e de pesquisa (incluindo aplicativos móveis) por mais de 30 anos [Sauro 2011]. Em seguida, as Entrevistas Semiestruturadas de Usabilidade (ESU) e o teste Think Aloud (TA), ambas usadas em 2 estudos.

Os métodos menos utilizados, citados em apenas 1 artigo, foram: o Teste de Usabilidade (TU), Questionário de Usabilidade (QU), Avaliação de Eficácia (AE), 
Questionário de Autoavaliação de Experiência do Usuário (QAEU), Entrevistas Semiestruturadas de Experiência do Usuário (ESUX), Questionário de Experiência do Usuário (QEU), Cognitive Walkthrough (CW) e Satisfação de Experiência do Usuário (SUX).

Ressalta-se que alguns estudos usaram mais de um método para avaliação, conforme descrito na etapa anterior (Subseção 3.1). Além disso, percebe-se que apenas o SUS, TA e CW, são métodos mais criteriosos de avaliação, inclusive, já consolidados na área de Interação Humano-Computador. As outras metodologias são menos formais, abrangendo características específicas do aplicativo, ficando a critério do avaliador, como por exemplo, as entrevistas semiestruturadas, que se baseavam nas funcionalidades do próprio produto.

\subsection{Perfil do Público-alvo dos Aplicativos}

As respostas para a segunda questão de pesquisa são mostradas na Figura 2, apresentando o público-alvo dos aplicativos.

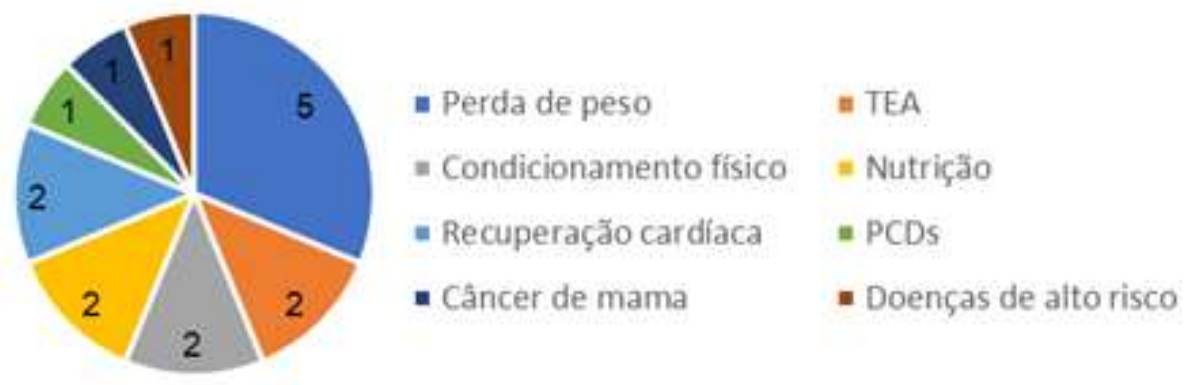

Figura 2. Público-alvo dos aplicativos

Usuários obesos e com sobrepeso são o público mais visado pelos aplicativos, com 5 ferramentas desenvolvidas. Posteriormente, usuários com TEA, praticantes de atividade física que buscavam melhorar o condicionamento físico, suporte à nutrição e pacientes em recuperação de alguma doença cardíaca, alcançaram o segundo lugar, com 2 aplicativos cada. Além disso, os PCDs, pacientes em tratamento do câncer de mama e doenças de alto risco, tiveram um aplicativo desenvolvido para cada.

\subsection{Etapa do Desenvolvimento que os Aplicativos foram Avaliados}

Com relação à terceira pergunta de pesquisa, apresentada na Figura 3, investigou-se qual etapa do desenvolvimento de software o aplicativo foi avaliado.

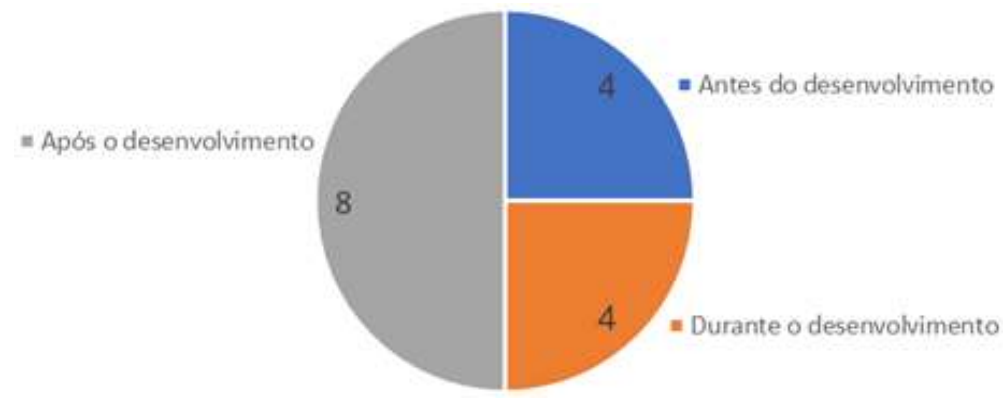

Figura 3. Etapa do desenvolvimento avaliada 
Observa-se que a somatória dos estudos totaliza 16, pois no estudo de [Alturki e Gay 2019], os testes com aplicativos foram realizados nas 3 fases de desenvolvimento.

A validação do software proporciona identificar facilidades e/ou dificuldades dos usuários na execução de tarefas do produto, antes do mesmo adentrar no mercado. Sendo assim, em 8 ocasiões (equivalente a 50\%), constituindo a maioria, as avaliações de usabilidade e/ou experiência do usuário foram realizadas na fase de implementação e/ou consolidação do aplicativo. Entretanto, quando são encontrados problemas (principalmente nas fases finais de desenvolvimento), há necessidade de retornar às etapas anteriores do projeto, acarretando uma perda de tempo preciosa na entrega do produto final ao cliente. Ademais, erros encontrados no software podem possibilitar uma experiência desagradável, ou seja, o usuário pode não ter estímulos suficientes para usar a ferramenta. Logo, uma boa estratégia para dispor aplicações eficazes e eficientes, é realizar as avaliações antes ou durante o período de desenvolvimento (em conjunto com os possíveis usuários), conforme apontou os outros $50 \%$ dos estudos (citado 4 vezes em ambas as fases); o feedback fornecido durante estas etapas contribui para o sucesso da aplicação, permitindo interfaces coesas e satisfação aos usuários.

\section{Considerações Finais}

Este estudo apresentou um Mapeamento Sistemático da Literatura, com o objetivo de proporcionar uma visão geral a respeito da investigação dos métodos de avaliação de usabilidade e UX de aplicativos móveis direcionados à prática de exercícios físicos. Contudo, pode ser que estudos relevantes não tenham sido incluídos, devido à quantidade limitada de bases de dados consultadas e a string de busca utilizada. Outra limitação desse estudo, foi que o protocolo não seguiu recomendações da área de saúde, e sim da computação. Também não se realizou a avaliação das metodologias dos estudos selecionados. Mas, apesar dessas limitações, os resultados mostraram que os testes de validação do software permitem elaborar ferramentas cada vez mais aptas a suprir às especificidades dos usuários, possibilitando uma experiência agradável, causando sensações e estímulos que fomentem a vontade de uso contínuo do aplicativo.

Além disso, aplicativos móveis para atividade física auxiliam o usuário/praticante a ser mais ativo, causando intervenções positivas para se ter hábitos saudáveis, qualidade de vida e bem-estar físico e mental; proporcionando dicas essenciais de nutrição para fomentar uma alimentação mais adequada e uma rotina diária de exercícios nesses indivíduos, diminuindo os níveis de sedentarismo e, reabilitando e prevenindo diversas doenças.

Os estudos selecionados, mediante ao mapeamento, enfatizaram que, o questionário SUS é um método comumente utilizado para avaliar a usabilidade de aplicativos e, sendo usado, em sua maioria, na etapa de implementação do aplicativo. Destaca-se, também, os métodos think aloud e cognitive walkthrough, e os avanços significativos na área de avaliação de UX, como a identificação e reutilização de métodos consolidados e validados.

Por fim, como etapas futuras, a pesquisa será replicada utilizando protocolos amplamente empregados na área de saúde como o Preferred Reporting Items for Systematic review and Meta-Analysis Protocols (PRISMA-P), ademais, o protocolo de estudo será registrado no site International Prospective Register of Systematic Reviews 
(PROSPERO), que proporciona respaldo para revisões sistemáticas. Também será realizada a avaliação das metodologias dos estudos selecionados, usando a ferramenta da Joanna Briggs Institute. No mais, buscar-se-á definir e disponibilizar um Guia de Recomendações de Usabilidade, integrando as melhores práticas dos métodos SUS, think aloud e cognitive walkthrough; esse guia será disponibilizado por meio de um site informativo, para apoiar o desenvolvimento de aplicativos móveis pertinentes à prática de exercícios físicos. Espera-se auxiliar os desenvolvedores na criação ou reengenharia dessas ferramentas, onde as mesmas sejam mais eficazes quanto ao seu objetivo, sendo aderentes às reais necessidades do seu público-alvo.

\section{Referências}

ACM (2021). "Digital Library", https://www.acm.org/publications/digital-library, Fevereiro.

Almeida, R. L., Darin, T., Viana, W., e Rossana, M. C. (2016). Um Mapeamento Sistemático sobre Avaliação de Modelos Mentais e Conceituais de Interfaces Digitais. Em Nuevas Ideas en Informática Educativa, páginas 142-151.

Alturki, R., e Gay, V. (2019). The development of an arabic weight-loss app akser waznk: Qualitative results. Em JMIR formative research, vol. 3, n. 1, páginas 1-20.

Aquino, E. M., Silveira, I. H., Pescarini, J. M., Aquino, R., e Souza-Filho, J. A. D. (2020). Social distancing measures to control the COVID-19 pandemic: potential impacts and challenges in Brazil. Em Ciencia \& saude coletiva, vol. 25, páginas 2423-2446.

Baig, M. M., GholamHosseini, H., and Ahmad, F. (2020) "A Usability and UserExperience Analysis of VitalsAssist: A mHealth Application to Monitor Vital Signs in Acute Care Settings", Annual International Conference of the IEEE Engineering in Medicine \& Biology Society (EMBC), p. 5132-5135.

Beatty, A. L., Magnusson, S. L., Fortney, J. C., Sayre, G. G., e Whooley, M. A. (2018). VA FitHeart, a mobile app for cardiac rehabilitation: usability study. Em JMIR human factors, vol. 5, n. 1, páginas 1-10.

Choo, S., Kim, J. Y., Jung, S. Y., Kim, S., Kim, J. E., Han, J. S., e Steinhubl, S. (2016). Development of a weight loss mobile app linked with an accelerometer for use in the clinic: usability, acceptability, and early testing of its impact on the patient-doctor relationship. Em JMIR mHealth and uHealth, vol. 4, n. 1, páginas 1-16.

Fonteyn, M. E., Kuipers, B., e Grobe, S. J. (1993). A description of think aloud method and protocol analysis. Em Qualitative health research, vol. 3, n. 4, páginas 430-441.

ISO/IEC 25000 (2011). "Software engineering -- Product quality", http://iso25000.com/index.php/en/iso-25000-standards/iso-25010, Março.

Jones, S., Keane, A., Stawiarski, A., Fatus, R., and Kane, B. (2016) "The impact of application system messages on the usability of healthcare software applications", International Symposium on Computer-Based Medical Systems (CBMS), p. 306-311. 
Kairy, D., Lehoux, P., Vincent, C., e Visintin, M. (2009). A systematic review of clinical outcomes, clinical process, healthcare utilization and costs associated with telerehabilitation. Em Disability and rehabilitation, vol. 31, n. 6, páginas 427-447.

Kanstrup, A. M., Bertelsen, P. S., Nunez, H. C., Jonasen, T. S., e Stage, J. (2018). MOVE: a mobile app designed for social health relations in residential areas. Em Building Continents of Knowledge in Oceans of Data: The Future of Co-Created eHealth, páginas 496-500.

Kim, B., Lee, D., Min, A., Paik, S., Frey, G., Bellini, S., e Shih, P. C. (2020). PuzzleWalk: A theory-driven iterative design inquiry of a mobile game for promoting physical activity in adults with autism spectrum disorder. Em Plos One, vol. 15, n. 9, páginas 1-24.

Kyu, H. H., Bachman, V. F., Alexander, L. T., Mumford, J. E., Afshin, A., Estep, K., e Forouzanfar, M. H. (2016). Physical activity and risk of breast cancer, colon cancer, diabetes, ischemic heart disease, and ischemic stroke events: systematic review and dose-response meta-analysis for the Global Burden of Disease Study 2013. Em The $B m j$, vol. 354 .

Lai, B., Wilroy, J., Young, H. J., Howell, J., Rimmer, J. H., Mehta, T., e Thirumalai, M. (2019). A mobile app to promote adapted exercise and social networking for people with physical disabilities: usability study. Em JMIR formative research, vol. 3, n. 1, páginas 1-11.

Lear, S. A., Hu, W., Rangarajan, S., Gasevic, D., Leong, D., Iqbal, R., e Yusuf, S. (2017). The effect of physical activity on mortality and cardiovascular disease in 130 000 people from 17 high-income, middle-income, and low-income countries: the PURE study. The Lancet, vol. 390, n. 10113, páginas 2643-2654.

Lee, D., Frey, G. C., Min, A., Kim, B., Cothran, D. J., Bellini, S., e Shih, P. C. (2020). Usability inquiry of a gamified behavior change app for increasing physical activity and reducing sedentary behavior in adults with and without autism spectrum disorder. Em Health Informatics Journal, vol. 26, n. 4, páginas 2992-3008.

Levin, M. E., Krafft, J., Seifert, S., e Lillis, J. (2020). Tracking valued and avoidant functions with health behaviors: A randomized controlled trial of the acceptance and commitment therapy matrix mobile app. Em Behavior Modification, páginas 1-27.

Lieffers, J. R., Arocha, J. F., Grindrod, K., e Hanning, R. M. (2018). Experiences and perceptions of adults accessing publicly available nutrition behavior-change mobile apps for weight management. Em Journal of the Academy of Nutrition and Dietetics, vol. 118 , n. 2, páginas 229-239.

Monteiro-Guerra, F., Signorelli, G. R., Tadas, S., Zubiete, E. D., Romero, O. R., Fernandez-Luque, L., e Caulfield, B. (2020). A Personalized Physical Activity Coaching App for Breast Cancer Survivors: Design Process e Early Prototype Testing. Em JMIR mHealth and uHealth, vol. 8, n. 7, páginas 1-22.

Petersen, K., Vakkalanka, S., e Kuzniarz, L. (2015). Guidelines for conducting systematic mapping studies in software engineering: An update. Em Information and Software Technology, vol. 64, páginas 1-18. 
Polson, P. G., Lewis, C., Rieman, J., e Wharton, C. (1992). Cognitive walkthroughs: a method for theory-based evaluation of user interfaces. Em International Journal of man-machine studies, vol. 36, n. 5, páginas 741-773.

PubMed (2021). https://pubmed.ncbi.nlm.nih.gov/about/, Fevereiro.

Romeo, A., Edney, S., Plotnikoff, R., Curtis, R., Ryan, J., Sanders, I., e Maher, C. (2019). Can smartphone apps increase physical activity? Systematic review and meta-analysis. Em Journal of medical Internet research, vol. 21, n. 3, páginas 1-14.

Sankaran, S., Dendale, P., e Coninx, K. (2019). Evaluating the impact of the HeartHab app on motivation, physical activity, quality of life, and risk factors of coronary artery disease patients: multidisciplinary crossover study. Em JMIR mHealth and uHealth, vol. 7, n. 4, páginas 1-14.

Sauro J. (2011). "Measuring usability with the system usability scale (SUS)", https://measuringu.com/sus/, Março.

Tong, H. L., Coiera, E., Tong, W., Wang, Y., Quiroz, J. C., Martin, P., e Laranjo, L. (2019). Efficacy of a mobile social networking intervention in promoting physical activity: Quasi-experimental study. Em JMIR mHealth and uHealth, vol. 7, n. 3, páginas 1-14.

Väätäjä, H., Koponen, T., and Roto, V. (2009) "Developing practical tools for user experience evaluation: a case from mobile news journalism", European Conference on Cognitive Ergonomics: Designing beyond the Product---Understanding Activity and User Experience in Ubiquitous Environments, p. 1-8.

Vermeeren, A. P., Law, E. L. C., Roto, V., Obrist, M., Hoonhout, J., and VäänänenVainio-Mattila, K. (2010) "User experience evaluation methods: current state and development needs", Proceedings of the 6th Nordic conference on human-computer interaction: Extending boundaries, p. 521-530.

Wang, J. B., Cataldo, J. K., Ayala, G. X., Natarajan, L., Cadmus-Bertram, L. A., White, M. M., e Pierce, J. P. (2016). Mobile and wearable device features that matter in promoting physical activity. Em Journal of mobile technology in medicine, vol. 5, n. 2 , páginas 1-10.

Wicahyono, G., Setyanto, A., Raharjo, S., and Munandar, A. (2019) "Pregnancy monitoring mobile application user experience assessment", International Conference on Information and Communications Technology (ICOIACT), p. 872-877.

Zhang, X., e Xu, X. (2020). Continuous use of fitness apps e shaping factors among college students: A mixed-method investigation. Em International journal of nursing sciences, vol. 7, páginas 80-87. 